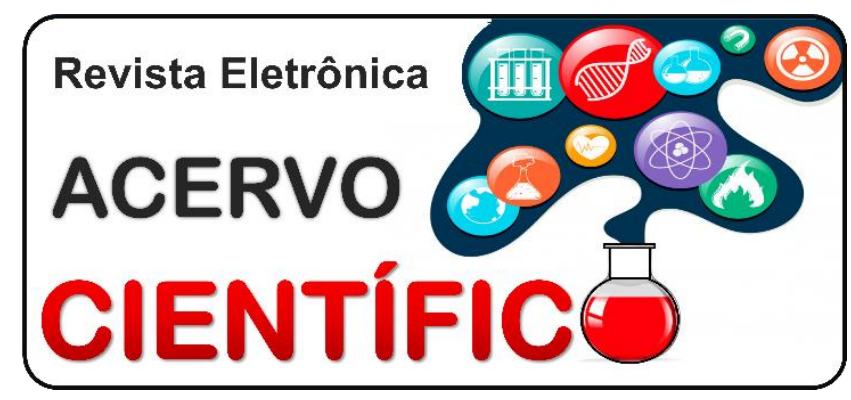

REVISÃO BIBLIOGRÁFICA

Recebido em: 8/2020

Aceito em: $7 / 2020$

Publicado em: 12/2020

\title{
A influência dos aspectos biopsicossociais nas elevadas taxas de suicídio da população transgênero
}

\author{
The influence of biopsychosocial aspects on the high suicide rates of the transgender \\ La influencia de los aspectos biopsicosociales em las altas tasas de suicídio de la \\ población transgénero
}

Amanda Rodrigues Rios ${ }^{1 *}$, Gustavo Fonseca Genelhu Soares ${ }^{1}$, Amanda Garcia da Costa ${ }^{1}$, Breno Artuso Lage ${ }^{2}$, Emanuelle Sousa Coutinho ${ }^{1}$, Guilherme Vinício de Sousa Silva ${ }^{3}$, Leonardo Antonio Vieira Lisboa ${ }^{1}$, Mariana Olympio Rua ${ }^{4}$, Matheus Rodrigues da Silva ${ }^{1}$, Thiago Pereira Soares ${ }^{5}$.

\begin{abstract}
Resumo: Esse artigo buscou analisar como os aspectos biopsicossociais específicos da população transgênero, travesti e não-binária influenciam nas elevadas taxas de suicídio desse grupo. Foram analisados como fatores de risco para o comportamento suicida nessa população o desrespeito ao nome social, dificuldade de acesso à terapia hormonal e cirurgias de adequação de gênero, rejeição familiar, disforia de gênero, trans/travestifobia, além do racismo dentro da mesma população. Ademais, observa-se a marginalização social dessa comunidade no acesso à atenção à saúde, diante de situações de discriminação, hostilidade e diagnóstico patologizante no processo transexualizador. Os resultados apontam aspectos biopsicossociais específicos da população transgênero, travesti e não-binária como fatores de risco consolidados para o suicídio, promovendo uma reflexão quanto à necessidade da elaboração de medidas de proteção a esses grupos, assim como a introdução desse tema nos diversos níveis da educação, além do fomento de pesquisas sobre o assunto, capacitação das equipes de saúde quanto à humanização e respeito às diferentes identidades de gênero.
\end{abstract}

Palavras-chave: Pessoas transgênero, Suicídio, Disforia de gênero.

\begin{abstract}
This article aimed to analyze how the specific biopsychosocial aspects of the transgender, transvestite and non-binary population influence the high suicide rates of this group. Disrespect for the social name, difficulty in accessing hormonal therapy and gender adequacy surgeries, family rejection, gender dysphoria, trans/travestiphobia, as well as racism within the same population were analyzed as risk factors for suicidal behavior in this population. In addition, it is observed the social marginalization of this community in access to health care, faced with situations of discrimination, hostility and pathological diagnosis in the gender reassignment process. The results point to specific biopsychosocial aspects of the transgender, transvestite and non-binary population as consolidated risk factors for suicide, promoting a reflection on the need to develop protective measures for these groups, as well as the introduction of this theme at different education stages, in addition to fostering research on the subject, training health staff on humanization and respect for different gender identities.
\end{abstract}

Keywords: Transgender persons, Suicide, Gender dysphoria.

${ }^{1}$ Centro Universitário de Caratinga (UNEC), Caratinga - MG. *E-mail: amandarios17@hotmail.com

2Universidade Federal dos Vales do Jequitinhonha e Mucuri (UFJVM), Teófilo Otoni - MG.

3Universidade Federal da Fronteira Sul (UFFS), Chapecó - SC.

${ }^{4}$ Universidade Vila Velha (UVV), Vila Velha - ES.

5Universidade de Marília (UNIMAR), Marília - SP. 
Resumen: Este artículo he buscado analizar cómo los aspectos biopsicosociales específicos de la población de transgénero, travestí y no binario influyen en las altas tasas de suicidio de este grupo. Se analizaron como factores de riesgo para la conducta suicida en esta población, la ausencia de respeto al nombre social, la dificultad para acceder a la terapia hormonal y a las cirugías de adecuación o cambio de género, el rechazo familiar, la disforia de género, la trans/travestibilidad y el racismo dentro de la misma población. Además, se puede observar la marginación social de esta comunidad en el acceso a la atención sanitaria, por situaciones de discriminación, hostilidad y diagnóstico patológico en el proceso de transexualización. Los resultados señalan aspectos biopsicosociales específicos de la población transgénero, travestí y no binario como factores de riesgo consolidados para el suicidio, promoviendo una reflexión sobre la necesidad de desarrollar medidas de protección de estos grupos, así como la introducción de este tema en los distintos niveles de la educación, además de la promoción de la investigación sobre el tema, la capacitación de los equipos de salud en la humanización y el respeto a las diferentes identidades de género.

Palabras clave: Personas transgénero, Suicidio, Disforia de género.

\section{INTRODUÇÃO}

O termo transgênero é designado àqueles cuja identidade de gênero não corresponde ao que seria esperado pelo sexo biológico de nascimento, sendo um termo guarda-chuva que abrange pessoas travestis e transexuais (TUCKER RP, et al., 2018).

Historicamente, a população transgênero é marginalizada e estigmatizada, devido, sobretudo, às forças conservadoras que estereotipam essas pessoas e colocam como normal apenas a manifestação cisgênero, que ocorre quando a identidade de gênero - masculino e feminino - correspondem aos padrões esperados pelo sexo biológico de nascimento - macho e fêmea (PERELSON S, 2011).

Em consequência disso, o preconceito contra travestis e transsexuais se manifesta de diversas formas, deixando essas pessoas numa situação de vulnerabilidade social. A dificuldade ao acesso à saúde pela população trans, sobretudo quanto à aquisição de terapias hormonais e cirúrgicas para a mudança de sexo, bem como os obstáculos impostos para o uso de nome social e a falta de apoio familiar são apenas algumas das dificuldades que esses indivíduos enfrentam no cotidiano (CHEN R, et al., 2019; RUSSEL ST, et al., 2018).

Em decorrência deste contexto marcado pelo preconceito e apagamento social, estudos mostraram que a taxa de suicí-dio entre a população transgênero é elevada. No Brasil, um estudo conduzido por Corrêa FHM; et al. (2020); Distrito Federal, demonstrou que $73,7 \%$ dos entrevistados declararam ter pensamentos suicidas e $29,9 \%$ disseram já terem tentado suicídio.

Já na China, um estudo revelou que $56,4 \%$ dos transgêneros entrevistados sofreram com ideações suicidas e 16,1 \% tentaram suicídio (RUSEN C, et al., 2019). Por fim, um estudo estadunidense relatou que $61,3 \%$ dos indivíduos transgêneros e não-binários entrevistados reportaram ideação suicida, taxa a qual é 3 vezes maior entre transgêneros se comparado com cisgêneros, cuja taxa é de 20,0\% (EISENBERG ME, et al., 2017).

Diante desse contexto, o objetivo deste estudo é realizar uma revisão bibliográfica com os estudos presentes na literatura, analisando como os aspectos biopsicossociais específicos da população transgênero influenciam na taxa de suicídio desse grupo, promovendo uma reflexão sobre o tema.

\section{REVISÃO BIBLIOGRÁFICA}

A vida da população trans como um todo no Brasil têm sido marcadas pela violência, e discriminação, como atestado pela literatura. Pesquisas brasileiras sobre os processos de marginalização social no acesso dessa população à atenção integral em saúde apontam situações de preconceito e hostilidade nos serviços. São também referidas dificuldade de compreensão das orientações médicas, ausência de exames físicos e, principalmente, o não respeito ao nome social. Ainda que os serviços especializados sejam percebidos como mais qualificados, não são incomuns os relatos de discriminação nesses casos. Tem sido pontuada a 
necessidade de capacitação das equipes de saúde, visando à humanização e respeito às diferentes identidades de gênero e sexuais (CARVALHO M e CARRARA S, 2013).

Estudos realizados no exterior sobre barreiras no cuidado em saúde da população trans apresentam resultados bem semelhantes. As disparidades no atendimento das necessidades de cuidado dessa população ficam mais evidentes ao se compararem pessoas de diferentes grupos sociais. Rocon PC, et al. (2016), em uma pesquisa realizada na sede da Associação Capixaba de Redução de Danos (ACARD) apontou o desrespeito ao nome social, a transfobia e o processo patologizante são considerados os principais fatores dificultadores no acesso ao sistema de saúde. Sendo assim, a transfobia nos serviços de saúde e o processo patologizante são considerados os principais impedimentos ao acesso universal, integral e equânime pelos participantes desta pesquisa ao SUS. As terapias hormonais e as cirurgias de adequação (redesignação sexual, mamoplastia), apesar de não serem um desejo universal na população trans, ainda representam uma importante ferramenta para lidar com a disforia de gênero. Dessa maneira, apresentariam, teoricamente, um potencial de redução do sofrimento mental e, consequentemente, da ideação e comportamento suicidas.

Entretanto, estudos dos últimos anos apresentam conclusões divergentes a respeito da temática. Chen $\mathrm{R}$, et al. (2019), em um estudo com 1309 transgêneros de 32 províncias da China, ao avaliar os índices de ideação suicida em pacientes que buscam esses tratamentos, observaram um aumento dessas taxas em homens transsexuais, o que poderia ser explicado pelas diversas barreiras que são encontradas no sistema de saúde para que se consiga as terapias desejada. Além disso, discordando de pesquisas anteriores, relatam que não encontraram associação que sugira que estressores biológicos como hormônios e cirurgias aumentem o risco suicida da referida comunidade. Já Tucker RP, et al. (2018), em um trabalho feito nos EUA com 206 veteranos de guerra transgêneros, relata uma redução substancial da ideação suicida em pacientes submetidos à terapia hormonal associada à cirurgia de redesignação sexual e mamoplastia quando comparado com quem realizou apenas uma das medidas ou nenhuma delas. Também concluíram, inesperadamente, que não houve mudança significativa quando comparados transexuais que foram submetidos a uma ou duas das três opções de tratamento citadas com aqueles que não receberam nenhum suporte, entretanto tais achados podem ter ocorrido por vieses de seleção da amostra estudada.

Por fim, Bauer GR, et al. (2015) e Wilson EC, et al. (2015), estudos que contaram com 380 e 314 integrantes trans, de Ontario (Canadá) e São Francisco (EUA), respectivamente, observaram uma redução da chance suicida mediante qualquer intervenção médica cirúrgica ou hormonal, sendo que o primeiro grupo de pesquisadores também relata que, dentro do grupo que considera o suicídio, a taxa de atentado contra a vida é maior no grupo em transição de gênero. Outro aspecto importante da vida da população transgênero a ser considerado é a possibilidade de uso do nome social, definido na cartilha "Garantia da utilização do nome social para pessoas transexuais e travestis", publicada pelo Ministério do Desenvolvimento Social e Agrário em 2016, como a designação pela qual esse grupo se identifica e é reconhecida pela comunidade, sem, no entanto, alterar o registro civil do indivíduo. Apesar de ser garantido em âmbito administrativo federal pelo Decreto Presidencial ํㅡ 8727/2016, essa determinação ainda não é sistematicamente cumprida, e nem sempre se estende às outras esferas institucionais, como os setores privados e públicos estaduais e municipais.

Uma pesquisa realizada por Russel ST, et al. (2018), em três cidades norte-americanas, com a participação de 129 pessoas transsexuais e não-binário, avaliou o uso do nome social em quatro esferas: em casa, no trabalho, na escola e com os amigos. Esse estudo revelou uma queda de $29 \%$ nos índices de ideação suicida e $56 \%$ nos de comportamento suicida quando o nome social era utilizado em um dos contextos, e, quando usado em todas as quatro situações, essas taxas apresentavam os menores níveis. A partir desses dados, percebe-se a importância de políticas governamentais que incentivem e legitimem esse direito, e que conscientizem a população dos impactos dessa denominação na saúde mental das pessoas trans.

Um ponto crucial na análise dos índices de suicídio entre os indivíduos transgêneros está na relação com a com a família. Em um estudo realizado por Klein A, et al. (2016), onde foi pesquisado a relação da rejeição familiar com o risco de suicídio entre transgêneros nos Estados Unidos, Distrito de Columbia, Porto Rico, Guam e llhas Virgens Americanas, constatou que $45,6 \%$ dos indivíduos trans pesquisados apresentaram falta 
de apoio familiar de moderado a alto, ainda foi observado que uma rejeição moderada aumenta por volta de duas vezes as chances de suicídio, e que o alto nível de desprezo aumenta em cerca de três vezes as chances de suicídio.

Corroborando os dados anteriores, em um estudo publicado por Eisenberg ME, et al. (2017), onde foram pesquisados fatores de risco e protetivos na saúde de jovens trans em escolas públicas do estado de Minnesota, Estados Unidos, dentre eles a conexão familiar, demonstrou que a jovens transgêneros e nãobinários apresentaram uma conexão familiar menor quando comparado aos jovens cis (3,53 versus. 4,27), a pesquisa também relatou que $61,3 \%$ dos jovens transgêneros e não-binários já tiveram pensamentos suicidas (versus. $20 \%$ na população cis jovem), e quando se observa a tentativa de suicídio, nos jovens transgêneros e não-binários foi de $31 \%$ (versus. $7,1 \%$ em jovens cis), demonstrando desse modo, a associação entre a falta de apoio familiar e a propensão ao suicídio. Por fim, ratificando o exposto anteriormente, foi constatado em uma revisão realizada por Virupaksha HG, et al. (2016), que cerca de $62 \%$ dos transgêneros já relataram alguma forma de problema com a família, e condições como confrontos no âmbito familiar e a negação do gênero pelos parentes, foram fatores importantes para a concretização do suicídio entre pessoas transgêneros.

Tais dados validam que a rejeição familiar é um potencial determinante dos altos índices de suicídio na população transgênero. E apesar dos estudos analisados não elucidar como a falta de apoio familiar é capaz de influenciar o comportamento suicida em pessoas transgêneros, é consenso que o amparo dos familiares pode propiciar uma melhor autoestima, gerar maior resiliência nas vivências estruturais e na discriminação interpessoal e possibilitar uma maior estabilidade emocional para o enfrentamento de circunstâncias estressantes da vida. Esses fatores impactam diretamente na diminuição de prejuízos mentais para essa população o que acarreta um menor risco de suicídio ao longo da vida desse grupo (KLEIN A, et al., 2018; VIRUPAKSHA HG, et al., 2016).

A disforia de gênero é uma condição marcada pela extrema identificação pessoal com o gênero oposto, de forma que este sentimento leva muitas vezes a ansiedade, depressão e vontade de viver com um gênero diferente ao seu atribuído (KALTIALA-HEINO R, et al., 2018). A todos é designado no ato do nascimento um gênero que vai de acordo com o seu sexo, utilizando-se da genitália para tal designação: pessoas nascidas com pênis sendo designados meninos e pessoas nascidas com vaginas sendo designadas meninas (REINER WG, 2012), porém, pesquisas na área da antropologia, neurociência e psiquiatria nos mostram que tal correlação não seja necessariamente a imperante. Beauvoir S (1949) em seu livro O Segundo Sexo, Vol. 2: A experiência vivida nos apresenta o começo de uma discussão filosófica sobre o gênero (principalmente o feminino), dizendo: "Ninguém nasce mulher: torna-se mulher. Nenhum destino biológico, psíquico, econômico define a forma que a fêmea humana assume no seio da sociedade; é o conjunto da civilização que elabora este produto...", nos mostrando que ao nascer a criança não apresenta qualquer tipo de tendência inata ao pertencimento a determinado gênero, sendo necessário o convívio social para tal. Já a psicologia define identidade de gênero como: "Gênero com qual uma pessoa se identifica, que pode ou não concordar com o gênero que the foi atribuído quando de seu nascimento" (JESUS JG, 2012).

O termo "disforia de gênero" aparece pela primeira vez na quinta edição do Manual diagnóstico e estatístico de transtornos mentais (DSM-5), onde abarca todas as pessoas que possuem incongruências entre o gênero expresso e seu gênero designado. Os critérios diagnósticos podem variar entre crianças e adultos, mas todos tangem o fato da incongruência entre o gênero expresso e o atribuído. Outros critérios são: preferência por atividades colocadas como do gênero oposto, uso de roupas não conformativas com o gênero de desígnio e forte rejeição a sua anatomia sexual (DSM, 2013). Na prática, a disforia de gênero é, como dito anteriormente, uma condição de extremo sentimento de inadequação e ansiedade, sendo que diversos estudos apontam a relação inadequação ou disforia de gênero com depressão e ideações suicidas (AZEEM R, et al., 2019; ZELUF G, et al., 2018; PETERSON CM, et al., 2016). Crianças diagnosticadas com disforia de gênero exibem índices elevados de depressão, ansiedade e do controle de impulsos, ocorrendo o mesmo em adultos, incluindo-se abuso de substâncias ilícitas e também pessoas que apresentam disforia de gênero de início tardio podem apresentar maiores sofrimentos e ambivalência sobre a cirurgia de redefinição de sexo e de maiores insatisfações pós-cirurgia (SADOCK BJ, et al., 2017). 
Apesar de ocorrer a demasiado tempo, e de já existir certa redução dessa situação, a discriminação contra a população transsexual ainda influência de forma imperativa a saúde mental desses indivíduos, podendo levar ao suicídio. Podemos observar esse tipo de influência, por exemplo, em veteranos no serviço militar americano. Segundo Tucker RP (2019), um dos grandes fatores que levava os veteranos transgêneros a terem intenções suicidas ou realmente recorrerem a tentativa de suicídio, era a discriminação, pois, além do preconceito em si, esse fator ainda dificultava para que conseguissem moradias próprias e até mesmo cuidados médicos.

Ademais, é possível observar que, no Paquistão, esse estigma frente à sociedade também priva essa parcela da população, muitas vezes, de terem acesso à educação e empregos. Compactuando com esse tipo de exclusão, há uma maior ocorrência de doenças mentais, como a ansiedade e a depressão, o que leva à idealização suicida, ou o suicídio completo em alguns casos (AZEEM R, et al., 2019). Cenário semelhante de estigma social é observado na Índia, onde a discriminação sofrida pelos indivíduos transgênero os impede de obter educação, meios de sobrevivência e moradia adequados, fazendo com que esse grupo recorra à moradia em favelas, mendicância e trabalho sexual. Estas condições, associadas à falta de apoio social, alta prevalência do HIV e questões relacionadas à violência, colaboram para uma taxa de suicídio de cerca de $31 \%$ da população transgênero, ainda que a prevalência exata de suicídio entre transgêneros não seja documentada (VIRUPAKSHA HG, et al., 2016).

É possível notar que uma das grandes influências do comportamento transfóbico das maiorias, advém de uma sociedade baseada em essencialismo de gênero, ou seja, uma sociedade voltada para uma separação de gênero e que impõe uma identificação com apenas duas possibilidades: feminino e masculino. E, para se adaptar no ambiente em que estão inseridos, a população transgênero se apoia em métodos de enfrentamento e grupos de apoio, uma forma de luta contra a enorme discriminação ainda existente (EDWARDS LL, et al., 2019).

Ainda é importante expor que, dentro dessa parcela da população, os negros e indígenas estão mais propensos a terem pensamentos suicidas ou tentarem, realmente, o suicídio ou algum ato de autoflagelação ao longo da vida, ou seja, além da discriminação por sua identidade de gênero, ainda existe um paradigma em relação à sua raça. Verifica-se, também, que indivíduos mais jovens apresentam maiores taxas de ideação suicida, em especial pessoas entre 21 e 25 anos, das quais $88,7 \%$ já experienciaram pensamento suicida. No entanto, mesmo com esse resultado, a significante maioria ainda espera recorrer ao tratamento psicológico e psiquiátrico para lidarem com essas dificuldades e problemas (CORRÊA FHM, et al., 2019).

\section{CONSIDERAÇÕES FINAIS}

Dado o exposto, infere-se que a saúde mental da população transsexual é caracterizada por fatores inerentes ao indivíduo, além de processos sociodemográficos relacionados ao ambiente ao qual a pessoa se insere. Nesse estudo foram apontados fatores de risco que contribuem para o aumento do sofrimento psíquico em pacientes trans e para as altas taxas de ideação suicida e suicídio entre essa população. Foi evidenciado que fatores como rejeição familiar, realização ou não de intervenções clínicas e cirúrgicas e preconceito sofrido por essa população são algumas das causas que corroboram para a alta taxa de autoquíria. Tendo em vista as diversas particularidades na vivência desses indivíduos, faz-se necessário a elaboração de medidas de proteção a esses cidadãos nos vários âmbitos da sociedade, assim como a introdução desse tema nos diversos níveis da educação, além do fomento de pesquisas e informação sobre essa problemática pode se fazer útil na promoção de uma melhor saúde a essa população.

\section{REFERÊNCIAS}

1. AMERICAN PSYCHIATRIC ASSOCIATION. DSM-V: Manual Diagnóstico e Estatístico de Transtornos Mentais. 5 ed. São Paulo: Artmed, 2015

2. AZEEM R. Prevalence of Suicide Ideation and its Relationship with Depression among Transgender Population. Journal of the College of Physicians and Surgeons Pakistan, 2019. 
3. BAUER GR, et al. Factors impacting transgender patients' discomfort with their family physicians: a respondent-driven sampling survey. PLoS One 2015; 10:e0145046.

4. BAUER GR, et al. Intervenable factors associated with suicide risk in transgender persons: a respondent driven sampling study in Ontario, Canada. BMC Public Health, 2015; 15: 525.

5. BEAUVOIR S. O Segundo Sexo: A Experiência Vivida. 5 ed. Rio de Janeiro: Editora Nova Fronteira, 2015.

6. BRASIL, Ministério do Desenvolvimento Social e Agrário. Garantia da utilização do nome social para pessoas transexuais e travestis, 2016.

7. CARVALHO M, CARRARA S. Em direito a um futuro trans: contribuição para a história do movimento de travestis e transexuais no Brasil. Sex Salud Soc (Rio J.) 2013; 8:319-51.

8. CHEN R, et al. Suicidal ideation and attempted suicide among Chinese transgender persons: National population study. Journal of Affective Disorders, 2019; 245: 1126-1134.

9. CORREA FHM, et al. Pensamento suicida entre a população transgênero: um estudo epidemiológico. J. bras. psiquiatr., Rio de Janeiro, Jan. 2020.

10. EDWARDS LL, et al. Resilience Factors and Suicide Risk for a Sample of Transgender Clients. Family Process Institute. Colorado, 2019.

11. EISENBERG ME, et al. Risk and Protective Factors in the Lives of Transgender/Gender Nonconforming Adolescents. J Adolesc Health, 2017; 61(4):521-526.

12. JESUS JG. Orientações Sobre Identidade de Genero: Conceitos e Termos. Núcleo de Ensino, Extensão e Pesquisa em Gênero e Sexualidade, 2014.

13. KALTIALA-HEINO R, et al. Gender dysphoria in adolescence: current perspectives. Adolescent Health, Medicine and Therapeutics, Finland, 2018; 9: 31-41.

14. KLEIN A, GOLUB SA. Family Rejection as a Predictor of Suicide Attempts and Substance Misuse Among Transgender and Gender Nonconforming Adults. LGBT Health. 2016;3(3):193-199.

15. PERELSON S. Transexualismo: uma questão do nosso tempo e para nosso tempo. ÉPOS, 2011, 2(2), 1-9.

16. PETERSON CM, et al. Suicidality, Self-Harm, and Body Dissatisfaction in Transgender Adolescents and Emerging Adults with Gender Dysphoria. The American Association of Suicidology, 2016.

17. REINER WG. Gender Identity and Sex Assignment: A Reappraisal for the 21st Century. Pediatric Gender Assignment. Advances in Experimental Medicine and Biology, Boston, 2002; Vol 511.

18. ROCON PC, et al. Dificuldades vividas por pessoas trans no acesso ao Sistema Único de Saúde. Ciênc. saúde coletiva, Rio de Janeiro, ago, 2016; 21(8): 2517-2526.

19. RUSEN C, et al.Suicidal ideation and attempted suicide amongst Chinese transgender persons: National population study. Journal of affective disorders, 2019.

20. RUSSEL ST, et al. Chosen Name Use Is Linked to Reduced Depressive Symptoms, Suicidal Ideation, and Suicidal Behavior Among Transgender Youth. Journal of Adolescent Health, 2018; 63: 503-505.

21. SADOCK BJ, et al. Compendio de Psiquiatria: Ciencia do Comportamento e Psiquiatria Clínica. 11 ed. São Paulo: Artmed, 2017.

22. TUCKER RP, et al. Hormone therapy, gender affirmation surgery, and their association with recent suicidal ideation and depression symptoms in transgender veterans. Psychological Medicine, 2018; 48 (14): 1-8.

23. TUCKER RP. Suicide in Transgender Veterans: Prevalence, Prevention and Implications of Current Policy. Perspectives on Psychological Science, 2019; 1-17.

24. VIRUPAKSHA HG, et al. Suicide and Suicidal Behavior among Transgender Persons. Indian J Psychol Med. 2016; 38(6):505-509.

25. WILSON EC, et al. Connecting the Dots: Examining Transgender Women's Utilization of Transition-Related Medical Care and Associations with Mental Health, Substance Use, and HIV. Journal of Urban Health: Bulletin of the New York Academy of Medicine, 2015; 92 (1): 182-192.

26. ZELUF G. Targeted Victimization and Suicidality Among Trans People: A Web-Based Survey. LGBT Health, 2018. 